\title{
TAGUNG
}

\section{Das deutsch-französische Duo im Spiegel der Zeit - aktueller Impulsgeber oder überholte Allianz?}

\author{
Simon Stro $\beta *$
}

Seitdem mit der Bildung einer Europäischen Gemeinschaft für Kohle und Stahl in den 1950er Jahren die europäische Integration Fahrt aufnahm spielt die Zusammenarbeit zwischen Deutschland und Frankreich eine entscheidende Rolle in der Ausgestaltung und Entwicklung dieses Prozesses. Auf der Basis der Aussöhnung der beiden vormals verfeindeten Staaten, entfaltete sich die supranationale und intergouvernementale Zusammenarbeit der sechs Gründungsmitglieder. In der Folge trat das deutsch-französische Tandem immer wieder gemeinsam als Taktgeber und Motor der Integrationsentwicklung auf europäischer Ebene auf. Hierbei wurde die Rolle des Duos von anderen europäischen Staaten schon immer zwiespältig gesehen. Es variierten im Laufe der Jahre Perzeptionen von willkommener Begrüßung und Unterstützung der Initiativen des Tandems bis zu strikter Ablehnung einer als teilweise zu dominant empfundenen und zu Lasten der Interessen anderer Staaten der Europäischen Union gehenden, deutsch-französischen Führungsrolle.

Der Workshop „The Franco-German couple leadership malgré nous et vous? Franco-German relationship seen from the outside" hatte das Ziel die momentane Rolle des deutschfranzösischen Duos in der Europäischen Union nach ,Lissabon' zu erörtern und dies mit einer Bestandsaufnahme der Perzeptionen des Einflusses des Duos seitens weiterer Staaten der Europäischen Union zu verknüpfen. Wissenschaftler vielfältiger akademischer Disziplinen von Forschungsinstituten und Universitäten einer Großzahl von Mitgliedstaaten der Europäischen Union diskutierten

\section{The Franco-German couple - leadership malgré nous et vous? Franco-German relationship seen from the outside}

Conference jointly organized by the TransEuropean Policy Studies Association (TEPSA) and the project, ,THESEUS establishing a European society" in cooperation with the Institut für Europäische Politik e.V.

Brüssel, 17.-18. Februar 2011

Welcome address

Mirte VAN DEN BERGE, TEPSA Executive Director, Brussels

Anja THOMAS, Research Associate; Programme Director THESEUS; Jean Monnet Chair for Political Science, Department of Political Science and European Studies, University of Cologne

Introductive discussion: the Franco-German couple in the historical context of EU integration and its importance within the EU Institutions

Chair: Jean-Victor LOUIS, Honorary member of the TEPSA Board, Brussels

Joachim SCHILD, Professor, Chair in Comparative Government and Politics, University of Trier

Panel 1: the Franco-German couple and the future economic governance and fiscal coordination in the $\mathbf{E U}$

Chair: Brendan DONNELLY, Director, Federal Trust, London

Iain BEGG, Professorial Research Fellow, London School of Economics (LSE), London

Jean-Victor LOUIS, Honorary member of the TEPSA Board, Brussels

Ramūnas VILPIŠAUSKAS, Director, Institute of International Relations and Political Science, Vilnius

Shane FITZGERALD, Researcher, Institute of International and European Affairs (IIEA), Dublin

* Simon Stroß, M.A., Marie Curie Early Stage Researcher, Trans European Policy Studies Association (TEPSA), Brüssel. 
kontrovers die Programmpunkte und ihre $\mathrm{zu}$ den Themen in Beziehung stehenden aktuellen Forschungsvorhaben. Die einzelnen Panels konzentrierten sich insbesondere auf die Rolle und Perzeptionen der deutsch-französischen Kooperation in Politikfeldern, die aktuell im Fokus nationaler Europapolitik sowie der wissenschaftlichen und öffentlichen Debatte stehen. Hierbei wurden die Themen auswärtige Beziehungen, im Speziellen die Gemeinsame Außen- und Sicherheitspolitik (GASP) sowie Erweiterungsfragen, EuroKrise und ,economic governance', die Reform des Budgets der Europäischen Union sowie die Entwicklung der Rolle des deutschfranzösischen Tandems im Wandel der Zeit behandelt.

\section{Euro-Krise und ,economic governance}

In der Anfangsphase der Euro-Krise im Frühjahr 2010 wurden die unterschiedlichen finanz- und wirtschaftspolitischen Ansätze Frankreichs und Deutschlands deutlich sichtbar. Hinsichtlich ihrer Reformvorschläge zur Vermeidung des erneuten Auftretens von Problemen rund um die gemeinsame Währung in der Zukunft setzte die französische Regierung auf ihr Konzept einer, gouvernemente économique', basierend auf den Prinzipien einer aktiven politisch gelenkten Wirtschaftspolitik, der Möglichkeit makroökonomischer Koordination und institutionellen Reformen. Die deutsche Seite wiederum trat für, economic governance', fokussierend auf strikter Stabilitätsorientierung, verbindlichen Regeln und einer bestehenden Unabhängigkeit der Europäischen Zentralbank, ein. Während zu Beginn der Krise das deutsch-französische Duo eher zögerlich agierte, vertrat es später gemeinsam vornehmlich Reformvorschläge in Richtung einer „economic governance“, allerdings begleitet von einer klaren Betonung intergouvernementaler Verfahren. Die Teilnehmer waren sich einig, dass Deutschland in der Euro-Krise einen größeren Einfluss auf die Diskussion hatte als Frankreich. Die belgische Ratspräsidentschaft im ersten Halbjahr 2010 übte Zurückhaltung in dieser Hinsicht und
Panel 2: France and Germany and enlargement policy and external action

Chair: Gunilla HEROLF, Senior researcher, Stockholm International Peace Research Institute (SIPRI), Stockholm

Katrin BÖTTGER, Deputy Director, Institut für Europäische Politik (IEP), Berlin

Atila ERALP, Director, Center for European Studies (CES), Middle East Technical University (METU), Ankara

Elfriede REGELSBERGER, Deputy Director, Institut für Europäische Politik (IEP), Berlin

Michele COMELLI, Senior Fellow, Istituto Affari Internazionali (IAI), Rome

\section{Dinner Speech}

Roland SCHÄFER, Minister, Deputy Head of political affairs, Permanent Representation of Federal Republic of Germany to the EU, Brussels

Comments: Gaël VEYSSIERE, Head of Press Department, Permanent Representation of France to the EU, Brussels

Panel 3: France and Germany and the reform of the EU budget

Chair: Daniel VERNET, Editor-in-Chief 'Boulevard Extérieur' and former Director International Relations 'Le Monde', Paris

Kārlis BUKOVSKIS, Assistant Director, Latvian Institute of International Affairs (LIIA), Riga

Brendan DONNELLY, Director, Federal Trust, London

Mojmir MRAK, Professor, Faculty of Economics, University of Ljubljana

Zsuzsánna BIEDERMANN, Junior research fellow, Institute for World Economics of the Hungarian Academy of Sciences (IWE), Budapest

Roundtable: Franco-German coupleleadership over time

Chair: Gianni BONVICINI, Executive Vice President, Istituto Affari Internazionali (IAI), Rome Jean-Paul JACQUÉ, TEPSA Secretary General, Brussels

Wolfgang WESSELS, TEPSA Chairperson; Jean Monnet Chair Professor, Department of Political Science and European Studies, University of Cologne Renaud DEHOUSSE, Director, Centre d'études européennes de Sciences Po, Paris

Vít BENEŠ, Researcher, Institute of International Relations (IIR), Prague

\section{Concluding Remarks}

Wolfgang WESSELS, TEPSA Chairperson; Jean Monnet Chair Professor, Department of Political Science and European Studies, University of Cologne 
gab so dem Tandem die Möglichkeit, die Agenda betreffend möglicher Reformansätze zu bestimmen.

Im Workshop wurden des Weiteren die Perzeptionen der Rolle Deutschlands und Frankreichs in der Euro-Krise in ausgewählten Mitgliedstaaten der Europäischen Union beleuchtet. So nahmen beispielsweise die Iren, ein Land, das selbst mit am stärksten von der Krise betroffen war und nach wie vor ist, besonders die vornehmlich deutschen Forderungen nach einer Senkung des irischen Unternehmenssteuersatzes negativ wahr und machten diese Überlegungen für eine Verschärfung seiner Lage auf den internationalen Finanzmärkten mitverantwortlich. Obwohl in Irland die Führungsrolle des Duos generell begrüßt wird und als wichtig erachtet werde, das Reformen im Bereich ,economic governance" von ihm angestoßen werden, werden jedoch Bedenken hinsichtlich einer empfundenen Missachtung der Gemeinschaftsmethode und der supranationalen Institutionen der Europäischen Union geäußert. Zwischen anderen Mitgliedstaaten gehen die Meinungen teilweise noch weiter auseinander: Während zum Beispiel in Slowenien die Reformvorschläge Deutschlands und Frankreichs unterstützt werden und die Ansicht vertreten wird, dass insbesondere das Schuldenproblem angegangen werden muss, sind die Schweden generell eher skeptisch gegenüber den Vorschlägen des Tandems eingestellt.

Reform des EU-Budgets und die finanzielle Vorausschau 2014 bis 2020

Die nächsten zwei Jahre werden von einer Debatte über den zu verabschiedenden mehrjährigen Finanzrahmen des Budgets der Europäischen Union von 2014 bis 2020 geprägt sein. Hierbei müssen die Mitgliedstaaten möglichst einen Konsens über die generelle Höhe des Budgets sowie die Zuteilungen der einzelnen Haushaltsposten erzielen. Es werden insbesondere zwei Punkte kontrovers diskutiert: Die Gruppe der sogenannten ,Nettozahler ${ }^{\circ}$ (unter anderem bestehend aus
Deutschland, den Niederlanden und Schweden) verwahrt sich strikt gegen einen Anstieg des Budgets. Des Weiteren ist sich die Europäische Union alles andere als einig, inwiefern die wichtigen Budgetposten Gemeinsame Agrarpolitik und Regionalfonds reformiert werden sollen. Im Workshop wurde die Sichtweise lettischer Politiker thematisiert, die es schwer finden, eine stringente Position zur Problematik einzunehmen solange das deutsch-französische Duo noch keine klare Linie gefunden hat. Während hinsichtlich einer Reform der Gemeinsamen Agrarpolitik lettische Ansichten eher mit der Position Frankreichs übereinstimmen und es argumentiert wird, dass sie generell - wenn auch restrukturiert und angepasst - beibehalten werden soll, ist die Meinung in Bezug auf Kohäsionspolitik eher auf der deutschen Linie. In Ungarn hat man Verständnis für die Argumentation der ,Nettozahler ' hinsichtlich einer Deckelung des Budgets, sieht aber aufgrund dessen die Notwendigkeit für einschneidende Reformen, um neue Aufgaben der Union nach ,Lissabon', beispielsweise in den Bereichen Außenpolitik und Klimaschutz, angehen zu können. Im Allgemeinen lässt sich feststellen, dass das deutsch-französische Tandem dringende Reformen des Haushalts der Europäischen Union momentan blockiert und hierbei eher als Bremser denn als Antrieb der Union wirkt. Es wurde hinzugefügt, dass die britische Regierung in dieser Hinsicht dem Duo zur Seite steht. Daraus resultierend werden die drei einflussreichsten Staaten der Union aller Voraussicht nach die entscheidende Kerngruppe im anstehenden Verhandlungsprozess bilden.

\section{Gemeinsame Außen- und Sicherheitspolitik}

Mit dem Inkrafttreten des Vertrags von Lissabon am 1. Dezember 2009 wurden signifikante Umstrukturierungen der GASP der Europäischen Union angestoßen. Insbesondere die Einführung des Postens einer Hohen Vertreterin der Union für Außen- und Sicherheitspolitik und des ihr unterstellten Europäischen Auswärtigen Dienstes sind hier zu 
nennen. Der Workshop thematisierte die entscheidenden Impulse der deutsch-französischen Achse für die Einführung der oben genannten Neuerungen sowie der Möglichkeit zur Ständigen Strukturierten Zusammenarbeit und den Ausbau von qualifizierten Mehrheitsentscheidungen in der GASP. Zugleich wurde jedoch deutlich, inwiefern andere Akteure der Union es ebenso verstanden, den Fokus auf weitere Bereiche und Regionen zu legen. Hierbei können als Beispiele die Beziehungen der Union zu Russland, die Europäische Nachbarschaftspolitik und Missionen der Gemeinsamen Sicherheits- und Verteidigungspolitik in Afrika benannt werden. Es wurde deutlich, dass im Bereich der GASP das deutsch-französische Tandem nicht im selben Maße der entscheidende Impulsgeber ist, wie dies in anderen Politikbereichen der Europäischen Union zu beobachten ist. Die Wahrnehmung in anderen Mitgliedstaaten bestätigt diese These. Es erscheint vielmehr, dass insbesondere die wichtige Rolle des Vereinigten Königreichs in der GASP in Betracht gezogen werden muss. Zusammen mit Frankreich bildet Großbritannien speziell in Verteidigungsfragen ein eigenes Duo, was durch die Kooperation der beiden Staaten in diesem Feld infolge des Abkommens von Saint-Malo belegt werden kann. Zusätzlich kommt hinzu, dass Frankreich, Deutschland und das Vereinigte Königreich zunehmend international als eine Art Troika in GASP-Fragen auftreten; so geschehen beispielsweise in Verhandlungen mit dem Iran über dessen Atomprogramm. Während die anderen Mitgliedstaaten der Europäischen Union die Arbeit der Troika in diesem Fall akzeptieren und unterstützen, sehen viele Länder andere Arten von Kooperation, wie etwa das Weimarer Dreieck oder die oben genannte Zusammenarbeit Frankreichs und Großbritanniens im Verteidigungssektor, sehr skeptisch. So fürchtet Italien durch eine enge Kooperation der drei großen Mitgliedstaaten zunehmend marginalisiert zu werden, während zugleich eine Führungsrolle Deutschlands und Frankreichs alleine durchaus begrüßt wird. In diesem Zusammenhang wurden auch Konzeptionen und mögliche zu- künftige Entwicklungen der europäischen Integration, wie etwa Ideen eines „Kerneuropas" oder eines „Europe à la carte“, diskutiert. Zusammenfassend kann das deutsch-französische Tandem in der GASP als Motor ohne Monopol bezeichnet werden.

\section{Erweiterung der Europäischen Union}

Nach der großen Erweiterungsrunde des letzten Jahrzehnts, welches die Aufnahme von 12 neuen Mitgliedstaaten sah, ist momentan in einigen Staaten Europas eine sogenannte ,enlargement fatigue" beobachtbar. Dieser Umstand ist insbesondere im Fall Deutschlands und Frankreichs innenpolitischen Gründen geschuldet. Generell lässt sich sagen, dass die Positionen Frankreichs und Deutschlands im Laufe der letzten Jahre zunehmend eine konvergente Entwicklung nahmen. Während insbesondere Deutschland zuvor ein starker Verfechter der letzten Erweiterungsrunde war, hat sich der Enthusiasmus in der Bundesrepublik in dieser Hinsicht deutlich abgekühlt und an Frankreichs skeptischere Position angepasst. Die Veränderung wird in der Türkei mit groBer Besorgnis gesehen. Die Rolle des Tandems wird in dieser Hinsicht von türkischer Seite als den ganzen Erweiterungsprozess bremsend gesehen. Es muss betont werden, dass die Türkei die Meinung des deutschfranzösischen Duos als wesentlich wichtiger einschätzt als beispielsweise diejenige der Mitgliedstaaten Schweden oder Spanien, die hinsichtlich eines EU-Beitritts der Türkei generell positiv eingestellt sind aber auf europäischer Ebene nicht den politischen Einfluss Deutschlands und Frankreichs haben. Hinzu kommt die deutlich artikulierte Ablehnung eines Beitritts der Türkei zur Europäischen Union von den Regierungen Merkel und Sarkozy. Es wurde jedoch angemerkt, dass für den Moment im Verhandlungsprozess der möglichen Erweiterung die deutsch-französische Achse keinen entscheidenden Einfluss nehmen kann, da die Verhandlungen einen automatischen Charakter haben und durch die Europäische Kommission dirigiert und ausgeführt werden. 
Das deutsch-französische Duo im Wandel der Zeit

Der Workshop machte deutlich, dass die Rolle und Perzeptionen des deutsch-französischen Tandems in Fragen der europäischen Integration aus vielerlei Blickwinkeln betrachtet werden können. Je nachdem, ob der Schwerpunkt auf die Führungskraft, den Inhalt, die Entwicklung, akademische Sichtweisen oder Perzeptionen in anderen Mitgliedstaaten gelegt wird, sind ambivalente Wertungen möglich. Generell waren sich die Teilnehmer einig, dass die Führungsrolle Frankreichs und Deutschlands, besonders in der Zeit vor dem wegweisenden Vertrag von Maastricht Anfang der 1990er Jahre, das Geschehen in der Union maßgeblich beeinflusst hat. Der Einfluss des Duos nahm in den darauffolgenden Jahren in mancher Hinsicht ab, was seine Begründung vornehmlich in der kontinuierlichen Erweiterung der Europäischen Union von damals 12 auf heutzutage 27 Mitgliedstaaten hatte. Nichtdestotrotz bildet die langanhaltende, enge und belastbare Beziehung zwischen den beiden Staaten bis heute die erfolgreichste Allianz innerhalb der Union. Die Rolle Deutschlands und Frankreichs in vielen Politikfeldern ist nach wie vor entscheidend; insbesondere in denjenigen, die von eher intergouvernementalen Verhandlungsmustern geprägt sind. Dies brachte dem Duo in jüngster Zeit den Vorwurf aus anderen Mitgliedstaaten ein, zu stark auf intergouvernementale Methoden der Problemlösung zu setzen und dabei die sogenannte Gemeinschaftsmethode zu vernachlässigen; ein Vorwurf der speziell gegen das Verhalten des Duos im Rahmen der Euro-Krise erhoben wurde. Es kann jedoch ebenfalls argumentiert werden, dass dem Vorangehen des deutschfranzösischen Duos im Europäischen Rat eine Supranationalisierung folgen kann, wie in der Vergangenheit bereits häufiger geschehen.

In Betreff auf die zukünftige Entwicklung der deutsch-französischen Partnerschaft lassen sich drei Thesen aufstellen. Erstens: Aufgrund einer Pfadabhängigkeit im deutsch-französi- schen Verhältnis ist es ein Reflex beider Seiten, immer zuerst den Partner zu konsultieren. Zweitens: In der Europäischen Union der 27 Mitgliedstaaten brauchen neue Initiativen eine starke Allianz die sie voranbringt. Momentan ist hier keine stabile Alternative zum deutsch-französischen Tandem sichtbar. Drittens: Die Besonderheit der Kooperation der beiden Staaten besteht speziell darin, dass aufgrund ihrer häufig unterschiedlichen nationalen Interessen im Hinblick auf den europäischen Integrationsprozess, ein einmal erreichter Kompromiss des Duos in vielen Fällen ohne Weiteres von den anderen Mitgliedstaaten mitgetragen werden kann.

Die generelle Perzeption der deutsch-französischen Rolle in der europäischen Integration differiert deutlich zwischen den einzelnen Mitgliedstaaten der Europäischen Union. So ist man in der Tschechischen Republik prinzipiell, aus historischen Gründen sowie dem Eigenbild eines ,tschechischen Exzeptionalismus" entsprechend sehr skeptisch gegenüber den Absichten europäischer ,Großmächte“ eingestellt. Diese Wahrnehmung überträgt sich folgerichtig auch auf die Perzeption des deutsch-französischen Duos. Demgegenüber wird beispielsweise in Belgien die Führungsrolle Deutschlands und Frankreichs im Allgemeinen sehr positiv gesehen und die Meinung vertreten, dass die enge Kooperation der beiden Partner, besonders vor dem Hintergrund ihrer vorherigen jahrhundertelangen Feindschaft, für alle europäischen Nachbarn wünschenswert ist.

\section{Ein Tandem vor neuen Herausforderungen}

Zum Ende des Workshops wurde die kontroverse Frage aufgeworfen, ob das deutschfranzösische Duo heutzutage überhaupt noch Relevanz besitzt. Es wurde deutlich, dass vor allem in bestimmten Politikfeldern wie der GASP sowie in der alltäglichen Politikgestaltung der Europäischen Union, die insbesondere von den Brüsseler Institutionen geführt wird, eine Vormachtstellung der beiden Staaten nicht mehr ersichtlich ist. Dennoch ist im- 
mer wieder in Krisensituationen beobachtbar, wie Deutschland und Frankreich die Initiative ergreifen und Politikvorschläge liefern, welche später im Konsens der Union angenommen werden. Es bleibt festzuhalten, dass das Tandem nach wie vor eine bedeutende Rolle in der europäischen Integration einnimmt, wenn auch neue Herausforderungen heutzu- tage und in der Zukunft das Tandem zwingen werden, sich und ihre Beziehung fortwährend anzupassen. Die zukünftige Entwicklung wird zeigen, ob das Tandem dann wie im Falle der Reform des Haushalts der Europäischen Union eher defensiv den Status quo verteidigen oder nach erneuten Stresstests konstruktiv mit Vorschlägen voranschreiten wird.

\title{
Zentrale Politikfelder der EU
}

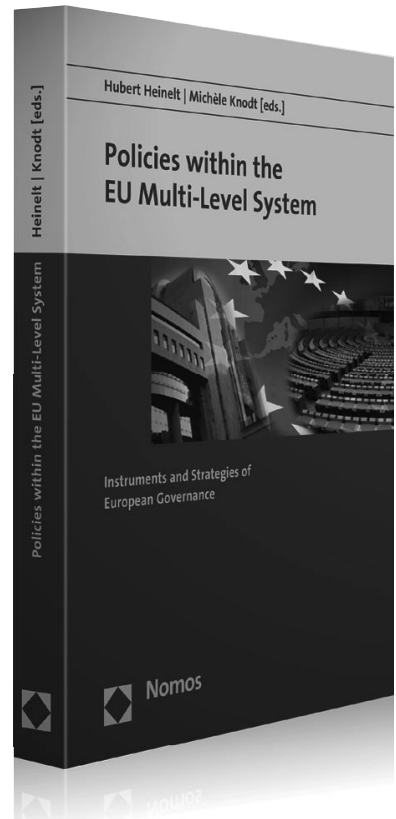

\author{
Policies within the \\ EU Multi-Level System \\ Instruments and Strategies \\ of European Governance
}

Herausgegeben von Prof. Dr. Hubert Heinelt und Prof. Dr. Michèle Knodt

2011, 410 S., brosch., 34, $-€$

ISBN 978-3-8329-6142-8

nomos-shop.de/13088

Dieser Sammelband gibt einen Überblick über die zentralen Politikfelder der EU. Dabei wird jeweils neben der historischen Entwicklung der einzelnen Politikfelder auf die wesentlichen Steuerungsmechanismen und die maßgeblichen Akteure bei der Politikformulierung und Implementation eingegangen und nach der konkreten Ausprägung und Bedeutung einer Mehrebenenstruktur gefragt.

Bitte bestellen Sie im Buchhandel oder versandkostenfrei unter $\bullet$ www.nomos-shop.de

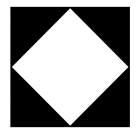

